\title{
Cleavage of proBDNF to BDNF by a Tolloid-Like Metalloproteinase Is Required for Acquisition of In Vitro Eyeblink Classical Conditioning
}

\author{
Joyce Keifer, Boris E. Sabirzhanov, Zhaoqing Zheng, Wei Li, and Timothy G. Clark ${ }^{\dagger}$ \\ Neuroscience Group, Division of Basic Biomedical Sciences, University of South Dakota School of Medicine, Vermillion, South Dakota 57069
}

\begin{abstract}
The tolloid/bone morphogenetic protein-1 family of metalloproteinases have an important role in the regulation of embryonic pattern formation and tissue morphogenesis. Studies suggest that they participate in mechanisms of synaptic plasticity in adults, but very little is known about their function. Recently, we isolated a reptilian ortholog of the tolloid gene family designated turtle tolloid-like gene $(t T l l)$. Here, we examined the role of tTLL in an in vitro model of eyeblink classical conditioning using an isolated brainstem preparation to assess its role in synaptic plasticity during conditioning. Analysis by real-time reverse transcription-PCR shows that an extracellularly secreted form of tTLL, tTLLs, is transiently expressed in the early stages of conditioning during conditioned response acquisition, whereas a cytosolic form, tTLLc, is not. Short interfering RNA (siRNA)-directed gene knockdown and rescue of tTLL expression demonstrate that it is required for conditioning. Significantly, we show that tTLLs cleaves the precursor proBDNF into mature BDNF in cleavage assay studies, and application of recombinant tTLLs protein alone to preparations results in induction of mature BDNF expression. The mature form of BDNF is minimally expressed in preparations treated with anti-tTLL siRNA, and the synaptic incorporation of both GluR1- and GluR4-containing AMPA receptors is significantly reduced, resulting in suppression of conditioning. This is the first study to demonstrate that expression of an extracellularly secreted tolloid-like metalloproteinase is regulated in the early stages of classical conditioning and functions in the conversion of proBDNF to mature BDNF. The mature form of BDNF is required for synaptic delivery of AMPA receptors and acquisition of conditioned responses.
\end{abstract}

\section{Introduction}

Neurotrophic factors including the tolloid/bone morphogenetic protein-1 (BMP-1) family of metalloproteinases enhance the survival, growth, and function of neurons. The tolloids and BMP-1 contain a conserved metalloproteinase domain not found in BMP2-BMP7, which are classed as transforming growth factor- $\beta$ (TGF $\beta$ ) superfamily members (Hopkins et al., 2007). The best characterized developmental response of the tolloid/BMP-1s is to regulate activity of TGF $\beta$ signaling. Recent studies suggest that growth factors activated during development may be induced later in adults during regulation of neuronal plasticity mechanisms (Lu et al., 2005), and members of the tolloid/BMP-1 family are implicated. An elevated number of hippocampal neurons expressing mammalian tolloid-like 1 (mTLL1) mRNA in mice was related to conditions that increase neurogenesis, such as access to running wheels (Tamura et al., 2005). Furthermore, a tolloid-like

Received July 28, 2009; revised 0ct. 6, 2009; accepted 0ct. 16, 2009.

This work was supported by National Institutes of Health Grants NS051187 and P20 RR015567, which is designated as a Center of Biomedical Research Excellence (J.K.). We thank Dr. Pat Ronan for helpful discussions throughout the course of this work and Drs. Ronan, Cliff Summers, and Brian Burrell for comments on this manuscript. We fondly remember our friend and colleague Dr. Tim Clark, who passed away during the course of this study.

'Deceased, Jan. 12, 2009

Correspondence should be addressed to Dr. Joyce Keifer, Neuroscience Group, Division of Basic Biomedical Sciences, University of South Dakota School of Medicine, 414 East Clark Street, Vermillion, SD 57069. E-mail: jkeifer@usd.edu.

DOI:10.1523/JNEUROSCI.3649-09.2009

Copyright $\odot 2009$ Society for Neuroscience $\quad$ 0270-6474/09/2914956-09\$15.00/0 gene, apTolloid/BMP-1, in the marine mollusk Aplysia was found to be regulated during nonassociative long-term sensitization (Liu et al., 1997). These studies suggest that tolloid/BMP-1 metalloproteinases have an important role in synaptic plasticity mechanisms as they do in normal patterning and development, but little is known about their function.

Previously, we characterized a reptilian ortholog of the tolloid gene family in pond turtles, tTll (turtle tolloid-like gene) (Sabirzhanov et al., 2007). Comparison of the amino acid sequence of tTLL revealed that it most closely resembles mammalian BMP-1 in its domain architecture and mTLL2 in its sequence. Interestingly, there are two transcripts, a secreted (tTLLs) and cytosolic (tTLLc) form. Given the established function of tolloid/BMP-1 in development and suggestive role in plasticity mechanisms, activity of tTLL in an in vitro model of classical conditioning was examined. A neural analog of eyeblink conditioning was studied using an isolated brainstem preparation from turtles (Keifer, 2003). Instead of using tone and air-puff stimuli as in behaving animals, we used paired stimulation of the auditory nerve [the "tone" conditioned stimulus (CS)] with the trigeminal nerve [the "air-puff" unconditioned stimulus (US)] and recorded burst discharge in the abducens nerve characteristic of a neural analog of blink responses. Synaptic delivery of GluR1- and GluR4-containing AMPA receptors (AMPARs) in abducens motor neurons supports conditioning in this preparation (Mokin et al., 2007; Zheng and Keifer, 2008, 2009) and brain-derived neurotrophic factor (BDNF) is critical (Li and Keifer, 2008, 2009a). 
Because formation of mature BDNF by the proteolytic conversion of the precursor proBDNF was implicated in long-term potentiation (LTP) (Pang et al., 2004; Lu et al., 2005) and because appearance of mature BDNF coincided with tTLL expression in our studies, we assessed whether tTLL functioned in cleavage of proBDNF during conditioning. Expression of the secreted splice variant tTLLs is significantly upregulated during conditioned response (CR) acquisition, and knockdown using anti-tTLL short interfering RNA (siRNA) indicate that it is required for conditioning. Significantly, tTLLs cleaves proBDNF into mature BDNF, which is a key signal transduction event required for synaptic AMPAR delivery during CR acquisition. These results are the first to identify a tolloid/BMP-1-like metalloproteinase that functions in the conversion of proBDNF to mature BDNF critical for synaptic plasticity underlying classical conditioning.

\section{Materials and Methods}

Conditioning procedures. Freshwater pond turtles, Pseudemys scripta elegans, obtained from commercial suppliers were anesthetized by hypothermia until torpid and decapitated. Protocols involving the use of animals complied with the guidelines of the National Institutes of Health and the Institutional Animal Care and Use Committee. The brainstem was transected at the levels of the trochlear and glossopharyngeal nerves, and the cerebellum was removed as described previously (Anderson and Keifer, 1999). The brainstem was continuously bathed in physiological saline $(2-4 \mathrm{ml} / \mathrm{min})$ containing $100 \mathrm{~mm} \mathrm{NaCl}, 6 \mathrm{~mm} \mathrm{KCl}, 40 \mathrm{~mm}$ $\mathrm{NaHCO}_{3}, 2.6 \mathrm{~mm} \mathrm{CaCl}_{2}, 1.6 \mathrm{~mm} \mathrm{MgCl}_{2}$, and $20 \mathrm{~mm}$ glucose, which was oxygenated with $95 \% \mathrm{O}_{2} / 5 \% \mathrm{CO}_{2}$ and maintained at room temperature $\left(22-24^{\circ} \mathrm{C}\right)$ at $\mathrm{pH}$ 7.6. Suction electrodes were used for stimulation and recording of cranial nerves. The US was an approximately twofold threshold single shock stimulus applied to the trigeminal nerve; the CS was a subthreshold $100 \mathrm{~Hz}, 1 \mathrm{~s}$ train stimulus applied to the ipsilateral auditory nerve that was below the threshold amplitude required to produce activity in the abducens nerve (Keifer et al., 1995; Anderson and Keifer, 1999). Neural activity was recorded from the ipsilateral abducens nerve that projects to the extraocular muscles controlling movements of the eye, nictitating membrane, and eyelid. The CS-US interval was $20 \mathrm{~ms}$, which is defined as the time between the offset of the CS and the onset of the US. This brief trace delay interval was found to be optimal for conditioning, but conditioning is not supported using longer trace intervals (Keifer, 2001). The intertrial interval between the paired stimuli was $30 \mathrm{~s}$. A pairing session consisted of 50 CS-US presentations (lasting $25 \mathrm{~min}$ in duration), followed by a $30 \mathrm{~min}$ rest period in which there was no stimulation (Keifer et al., 1995). Conditioned responses were defined as abducens nerve activity that occurred during the CS and exceeded an amplitude of double the baseline recording level. Conditioned preparations received paired CS-US stimulation, whereas pseudoconditioned controls received the same number of CS and US exposures that were explicitly unpaired using a CS-US interval randomly selected between $100 \mathrm{~ms}$ and $25 \mathrm{~s}$.

Design of siRNAs and application to in vitro preparations. Dicersubstrate duplex RNAs consisting of 27-mer with two base 3' overhangs (siRNA) were designed by RNA interference (RNAi) design software (Integrated DNA Technologies) for the tTLL target gene. The sense sequence was 5 '-GGACUGACGAGGAGAGCUUCAUUGT-3', and the antisense sequence was $5^{\prime}$-ACAAUGAAGCUCUCCUCGUCAGUCCUC-3'. The siRNA for tTLL was designed to bind with both transcripts of tTLL, tTLLs and tTLLc, at sites 575-599 and 686-710 bp, respectively, which are the same for both sequences (Sabirzhanov et al., 2007). Oligonucleotides containing the siRNA sequence were synthesized and purified by Integrated DNA Technologies. For negative controls, we used the Silencer siRNA Screening Control Panel, specifically Silencer Negative Control \#6 and \#7 (Ambion). Each of these negative control siRNAs were designed to have no significant homology to any known gene sequences from human, mouse, or rat species. The designed dicer-substrate duplex RNAs were checked by the basic local alignment search tool BLAST database (http://blast.ncbi.nlm.nih.gov/) to ensure unique targets. Turtle brainstem preparations ( $n$ values are reported in Results) were incubated with $100 \mathrm{~nm}$ anti-tTLL siRNA or Silencer Negative Control \#6 or \#7 mixed in Lipofectamine RNAiMax $(2.5 \mu \mathrm{l} / \mathrm{ml}$; Invitrogen) and oxygenated physiological saline at room temperature for $25 \mathrm{~h}$. Preparations were washed for $1 \mathrm{~h}$ in saline, followed by conditioning for two pairing sessions. After conditioning, they were frozen in liquid nitrogen for later analysis of tTLL expression by real-time reverse transcription (RT)-PCR.

tTLL siRNA resistant construct ( $t$ TLLs rescue plasmid) and application procedures. The sequence corresponding to the full-length variant of tTLLs and having a V5 epitope and polyhistidine region on the $\mathrm{C}$ terminus was inserted into a pCIG expression vector between HindIII and SmaI sites. The rescue plasmid was made by introducing three nucleotide mutations (GAG to TCA) to the binding region of the tTLL siRNA using the QuikChange Site-Directed Mutagenesis kit (Stratagene). This plasmid will express mRNA for tTLLs but not for tTLLc because of sequence differences at the $5^{\prime}$ end of the transcript. To test the function of the rescue plasmid in cell cultures, COS-1 Cercopithecus aethiops kidney cells were transfected with either tTLLs siRNA rescue plasmid, or tTLLs or tTLLc expression plasmid. After $8 \mathrm{~h}$, cells were transfected with the antitTLL siRNA (100 nM) using Lipofectamine RNAiMax. After incubation for $17 \mathrm{~h}$, cells were harvested, and expression levels for tTLLs and tTLLc were examined by Western blotting using anti-6X His antibodies (Abcam). For introduction of plasmids into brainstem preparations, a 0.135 pm concentration ( $1 \mu$ l total volume) of DNA rescue tTLLs pCIG was pressure microinjected into the auditory and abducens nerves, and preparations were incubated in physiological saline for $8 \mathrm{~h}$. This procedure allowed for selective transfection of presynaptic auditory nerve terminals that convey the CS and postsynaptic abducens motor neurons that convey the conditioned and unconditioned blink responses. After incubation, $100 \mathrm{~nm}$ anti-tTLLs siRNA was added to the bath, and preparations were incubated for an additional $17 \mathrm{~h}$, followed by wash and conditioning for two pairing sessions as described above. After the physiological experiments, tissue was frozen in liquid nitrogen and analyzed for tTLL expression by real-time RT-PCR.

Cell culture and transfection. COS-1 kidney cells and Mus musculus $\mathrm{NIH} / 3 \mathrm{~T} 3$ fibroblast cells were maintained in DMEM supplemented with $10 \%$ heat-inactivated fetal bovine serum, $100 \mathrm{U} / \mathrm{ml}$ penicillin, and 100 $\mu \mathrm{g} / \mathrm{ml}$ streptomycin at $37^{\circ} \mathrm{C}$ and $5 \% \mathrm{CO}_{2}$ atmosphere. Cells at $70 \%$ confluence were transfected with $15 \mu \mathrm{g}$ of tTLL expression vector. Plasmids $(15 \mu \mathrm{g})$ were diluted in $100 \mu \mathrm{l}$ of Opti-MEM I Reduced Serum Medium, and transfection of cells was performed using Lipofectamine LTX Reagent ( $25 \mu \mathrm{l}$; Invitrogen) incubated for $30 \mathrm{~min}$ at room temperature.

tTLL expression constructs. Sequences corresponding to full-length tTlls and $t$ Tllc cDNA (National Center for Biotechnology Information identification numbers FJ514825.1 and FJ514824.1, respectively) were inserted into a pcDNA3.1/V5-HisTOPO TA expression vector. The resulting recombinant proteins contain the $\mathrm{V} 5$ epitope and a polyhistidine region at the $\mathrm{C}$ terminus. We also created inactive $t$ Tlls and $t$ Tllc by mutation of Glu to Ala in the zinc binding consensus sequence HExxHxxGxxH. This amino acid sequence is conserved in all astacin-like metalloproteinases (Bode et al., 1992), and the HExxHxxAxxH mutant is inactive (Yiallouros et al., 2000).

Expression and purification of recombinant tTLL proteins. COS-1 cells were transfected with tTLL expression vectors. After $48 \mathrm{~h}$, cells (tTLLc) or medium (tTLLs) were harvested. Protease inhibitors were added to the cell lysates to final concentrations of $10 \mu \mathrm{g} / \mathrm{ml}$ leupeptin and $0.4 \mathrm{~mm}$ PMSF. The supernatant was centrifuged to remove debris and concentrated, and recombinant proteins were purified by using the ProBond Purification System (Invitrogen) under native conditions. Proteins were visualized by SDS-PAGE on $4-20 \%$ gradient gel and SYPRO Ruby protein gel staining.

Turtle proBDNF expression constructs and expression and purification of recombinant turtle proBDNF and its cleavage-resistant form. The fulllength cDNA sequence of turtle proBDNF was inserted into a Champion pET 101 Directional TOPO expression vector (Invitrogen). The expression constructs for production of cleavage-resistant proBDNF were also prepared by point mutations introduced into the proconvertase cleavage site of proBDNF converting the Arg-Arg to Ala-Ala. In vitro studies have 
Table 1. Primers and probes used for the analysis of tTLL by real-time RT-PCR

\begin{tabular}{llll}
\hline Target & Forward primer & Reverse primer & MGB probe \\
\hline Total tTIl & AGGCACGACAGTTGTGCTTATG & CAATCAAAGGGCTGTCCTCAGT & CTACTTGGAAATCCGAGACGGCCC \\
tTLLs & GGAGAGCTGGAGCAGCAA & CAAGTCCTCTTCGTCTAAAGCAATATC & TACCGCGACCCCTGCAAAGCC \\
tTLLC & TCGCCCAGGCTACCCATATG & TGGAGGCCCCTCAGTGCGC \\
Turtle actin & GGGGCAGTGGGCATTTCATCTC & AAGCTGTGCTATGTTGC \\
\hline
\end{tabular}

shown that proBDNF with this mutation is resistant to cleavage by furin and plasmin (Pang et al., 2004; Woo et al., 2005). BL21 Star(DE3) One Shot Escherichia coli cells were transformed with the proBDNF expression plasmids. Cells were harvested $6 \mathrm{~h}$ later under denaturing conditions. Protease inhibitors were added to the cell lysates to a final concentration $10 \mu \mathrm{g} / \mathrm{ml}$ leupeptin and $0.4 \mathrm{~mm}$ PMSF.

$B D N F$ precursor cleavage assay. Recombinant proBDNF protein or cleavage-resistant mutants of proBDNF (200 ng) were incubated alone or with purified recombinant tTLLs, tTLLc, mutant tTLLs, or mutant tTLLc (20 ng) in $50 \mathrm{~mm}$ Tris- $\mathrm{HCl}$, pH 7.5, $150 \mathrm{~mm} \mathrm{NaCl}$, and $5 \mathrm{~mm} \mathrm{CaCl}_{2}$ for $16 \mathrm{~h}$ at $37^{\circ} \mathrm{C}$. Reactions were stopped by adding SDS-PAGE loading buffer and boiling for $5 \mathrm{~min}$. Assay samples were subjected to SDS-PAGE on $4-20 \%$ gradient gels and analyzed by using anti-BDNF rabbit polyclonal antibody directed against mature BDNF (catalog \#20981; Santa Cruz Biotechnology). All reactions were performed twice.

Real-time RT-PCR. Total RNA was isolated from turtle brainstems using TRI Reagent (Molecular Research Center). Real-time RT-PCR was performed using $50 \mathrm{ng}$ of total RNA per reaction. RNA was combined with primer/probe sets and TaqMan Gold RT-PCR Master Mix (Applied Biosystems). Gene-specific primers and probes were created for P. scripta elegans to total tTLL, both splice variants tTLLs and tTLLc, and turtle actin, using the Primer Express Software (Applied Biosystems), and these are shown in Table 1 (Sabirzhanov et al., 2007). Real-time assays were run on an ABI PRISM 7000 analyzer (Applied Biosystems). The RT-PCR profile consisted of one cycle at $48^{\circ} \mathrm{C}$ for $30 \mathrm{~min}$ and $95^{\circ} \mathrm{C}$ for $10 \mathrm{~min}$, followed by 40 cycles at $95^{\circ} \mathrm{C}$ for $15 \mathrm{~s}$ and $60^{\circ} \mathrm{C}$ for $1 \mathrm{~min}$. The results of the real-time RT-PCR were normalized to turtle actin. Samples were confirmed to be free of DNA contamination by performing reactions without reverse transcriptase. All reactions were performed twice on each preparation. To obtain the fold change in mRNA, data were analyzed using the 2- $\Delta$ CT method (Livak and Schmittgen, 2001).

Glutamate receptor localization, confocal imaging, and data analysis. Immediately after the physiological experiments, brainstems were immersion fixed in cold $0.5 \%$ paraformaldehyde. Tissue sections were cut at $30 \mu \mathrm{m}$ and incubated in primary antibody overnight at $4^{\circ} \mathrm{C}$ with gentle shaking. The primary antibodies used were a polyclonal antibody raised in goat that recognizes the GluR4 subunit of AMPARs (1:100; catalog \#7614; Santa Cruz Biotechnology), a polyclonal raised in rabbit that recognizes GluR1 (1:200; catalog \#1504; Millipore Bioscience Research Reagents), and a monoclonal antibody raised in mouse that recognizes synaptophysin (1:1000; catalog \#5768; Sigma). After the primary antibodies, sections were rinsed and incubated with secondary antibodies for $2 \mathrm{~h}$ using a concentration of 1:100 for GluR4 and GluR1 or 1:200 for synaptophysin. The secondary antibodies were a cyanine 3 (Cy3)conjugated goat anti-rabbit IgG for GluR1, a Cy3-conjugated rabbit antigoat IgG for GluR4, and a Cy2-conjugated goat anti-mouse IgG for synaptophysin (Jackson ImmunoResearch) that were used to visualize the primary antibodies. Sections were rinsed, mounted on slides, and coverslipped. Images of labeled neurons in the principal or accessory abducens motor nuclei were obtained using an Olympus Fluoview 500 laser scanning confocal microscope. Tissue samples were scanned using a $60 \times 1.4$ numerical aperture oil-immersion objective with dual excitation using a $488 \mathrm{~nm}$ argon laser and a $543 \mathrm{~nm}$ helium-neon laser. Quantification of punctate staining of at least twofold greater intensity above background was performed using stereological procedures (Mokin and Keifer, 2006) with MetaMorph software (Universal Imaging Corporation). Images of two consecutive optical sections were taken using confocal microscopy. Protein puncta were counted in one optical section (sample section) if they were not present in the optical section immediately below the sample section (lookup section) and if they were within the inclusion boundaries of the unbiased counting frame. Colocalized staining indicating the presence of glutamate receptor subunits at synaptic sites was determined when red and green puncta were immediately adjacent to one another or if they were overlapping. Statistical analysis of the data was performed using a one-way ANOVA, followed by Fisher's and Bonferroni's tests for post hoc comparisons. $p$ values are indicated for specific comparisons in the text.

\section{Results}

Expression of a secreted but not a cytosolic form of tTLL mRNA is upregulated during in vitro classical conditioning Classical conditioning of a neural analog of eyeblink responses can be generated in an isolated brainstem preparation that contains the pontine blink circuitry (Keifer et al., 1995; Anderson and Keifer, 1999; Keifer, 2003; Zhu and Keifer, 2004). An electric shock US applied to the trigeminal nerve evokes a burst discharge recorded in the abducens nerve (Fig. $1 A$, top right taken before acquisition of CRs) that is characteristic of electromyographic recordings made during eyeblink movements in behaving turtles and in a reduced preparation during blinking (Keifer, 1993, 2003). Pairing of a CS to the auditory nerve before trigeminal nerve stimulation results in burst discharge that is representative of a CR (Fig. $1 A$, bottom right, arrow). The CRs are generally recorded by the beginning of the second pairing session after 50 paired stimulus presentations, followed by a brief rest period, or after $\sim 1 \mathrm{~h}$. An example of a typical acquisition curve taken from the current data is shown (Fig. $1 A$, left). During the first pairing session, there are usually a minimal number of CRs. However, by the beginning of the second pairing session, the percentage of CRs rapidly increases during acquisition that is followed by a period of asymptotic CR expression with additional pairing.

The timing of mRNA expression during conditioning for total tTLL and the individual splice variants tTLLc and tTLLs was examined by real-time RT-PCR, and these data are summarized in Figure $1 \mathrm{~B}$. Suitable antibodies that recognize the tolloid family of metalloproteinases are not available for the examination of protein and studies are currently limited to the mRNA. Levels of tTLLs expression were significantly increased by nearly 16 -fold after only one pairing session of conditioning or $25 \mathrm{~min}$ after the onset of paired stimulation compared with the pseudoconditioned group (Fig. $1 B, n=4$ preparations; $p<0.0001, \mathrm{C} 1$ vs Ps 1 ). In contrast, expression of tTLLc after one session of conditioning was unchanged from controls ( $p=0.99, \mathrm{C} 1$ vs Ps1). These RTPCR data correspond to physiological data obtained from the same preparations that averaged $25 \%$ CRs compared with $0 \%$ CRs after pseudoconditioning (Fig. $1 \mathrm{~A}$, left). The high level of tTLLs expression was maintained to $>13$-fold in preparations conditioned for two sessions or $80 \mathrm{~min}$ after the onset of paired stimulation ( $n=6$ preparations, $p<0.0001$, C2 vs Ps 2 ), when acquisition of CRs reached $69 \%$. This is in contrast to the unchanged values of tTLLc expression in the same preparations compared with pseudoconditioning ( $p=0.99, \mathrm{C} 2$ vs Ps2). By the fifth pairing session, or $4 \mathrm{~h}$ after the onset of conditioning, levels of tTLLs were observed to decline to control values ( $n=6$ preparations, $p=0.81$, C5 vs Ps5), although the percentage of CR 
A
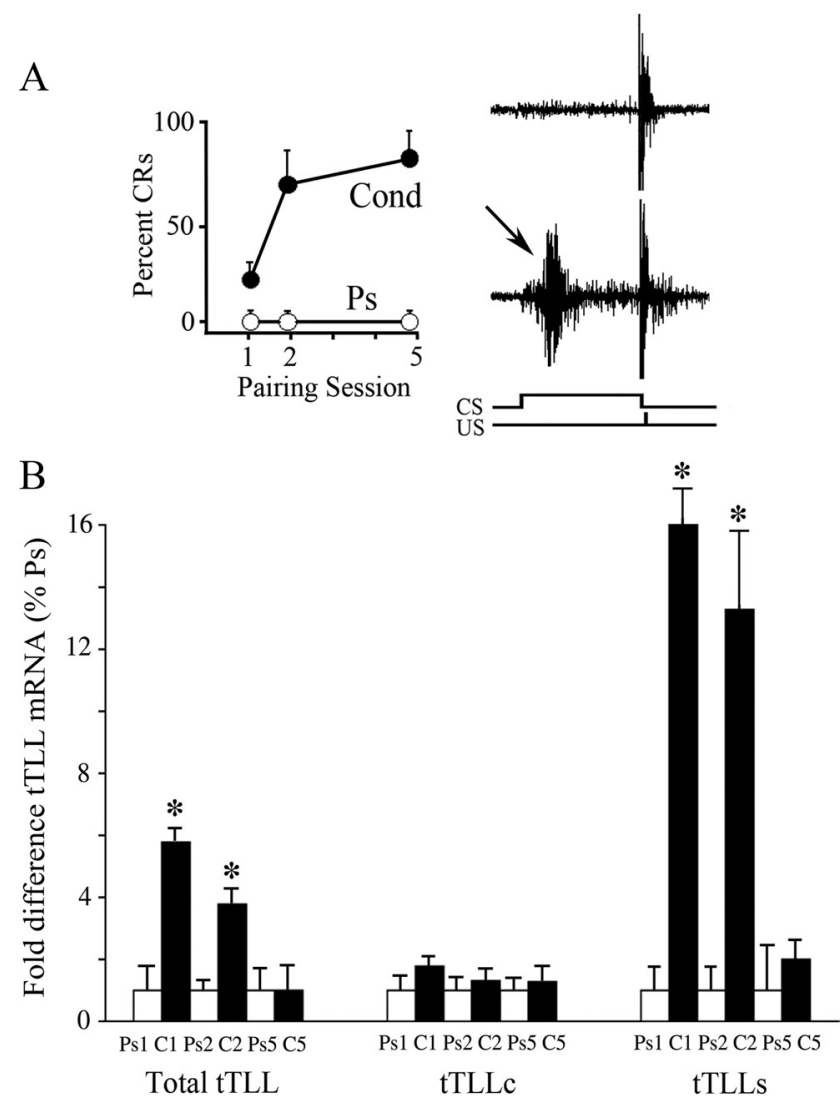

Figure 1. Expression of tTLLs MRNA is upregulated in the early stages of conditioning. $A$, Acquisition curves of the mean percentage of CRs recorded from the same preparations analyzed by RT-PCR that were conditioned (Cond) or pseudoconditioned (Ps) for one, two, or five pairing sessions. Examples of physiological records from the abducens nerve show an unconditioned response alone (top record) and a CR (arrow), followed by the unconditioned response after acquisition (lower record). The CS and US are illustrated at the bottom. $\boldsymbol{B}$, Real-time RT-PCR analysis of the total level of tTL mRNA and of the cytosolic (tTLLc) and secreted (tTLLs) splice variants expressed as the mean fold difference compared with pseudoconditioned levels. Data are shown from preparations that were conditioned or pseudoconditioned for one pairing session (Ps1, C1; or a total of $25 \mathrm{~min}$ ), two pairing sessions (Ps2, C2; or a total of $80 \mathrm{~min}$ ), or five sessions (Ps5, C5; or $\sim 4 \mathrm{~h}$ ). Data are plotted as means \pm SEM, except for conditioning data that are expressed as means \pm SD. Asterisks indicate significant differences from Ps for all figures.

expression was substantial at $81 \%$. Levels of tTLLc remained unchanged ( $p=0.99$, C5 vs Ps5). These data indicate that expression of the extracellularly secreted rather than the cytosolic form of tTLL mRNA, tTLLs, is selectively upregulated during conditioning. Moreover, expression of tTLLs is transient and occurs in the early stages of conditioning during CR acquisition but declines in later stages, although CR expression is high.

\section{Knockdown of tTLL expression by an anti-tTLL siRNA suppresses conditioning}

To selectively knock down tTLL mRNA expression to assess its function during conditioning, an RNAi approach was used. The siRNA oligonucleotides targeting tTLL were designed to bind to identical sequences shared by both splice variants tTLLs and tTLLc (Sabirzhanov et al., 2007). For control siRNA oligonucleotides, we used commercially available negative control siRNAs that do not target any known gene (Negative Control \#6 and \#7; Ambion). The ability of the anti-tTLL siRNA to knockdown tTLL expression was first assessed using an in vitro assay method in cell culture (Fig. 2A). COS1 cells were transfected with tTLLs, tTLLc,
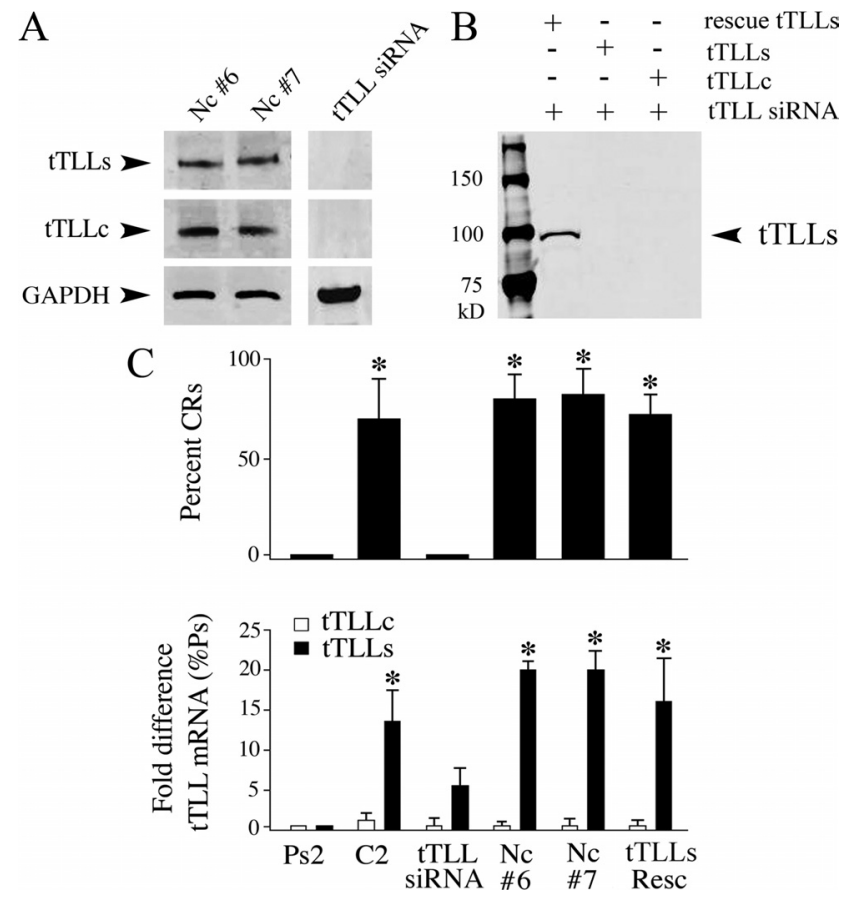

Figure 2. Treatment with anti-tTLL siRNA suppresses conditioning and is reversed by application of tTLLs rescue plasmids. $A$, The battery of siRNAs used here, Negative Control \#6 (Nc \#6), Negative Control \#7 (Nc \#7), and the anti-tTLL siRNA (tTLL siRNA), was tested for their effectiveness in knocking down target genes using cell cultures. COS-1 cells were transfected with tTLLs, tTLLC, or tGAPDH expression plasmids and later transfected with one of the siRNAs and examined by Western blot. The Nc \#6 and Nc \#7 siRNAs had no effect on expression of either splice variant of tTLL or tGAPDH. The anti-tTLL siRNA effectively blocked expression of both tTLLs and tTLLc but had no effect on TGAPDH. $B$, COS-1 cells were transfected with a tTLLs rescue plasmid that was rendered resistant to the anti-tTLL siRNA by a mutation in the binding region of the siRNA. Cells were transfected with rescue tTLLs, or unmutated tTLLs or tTLLc expression plasmids and then transfected with anti-tTLL siRNA. Western blots showed that tTLL was expressed only by cells that were transfected by the rescue plasmid demonstrating resistance to the anti-tTLL siRNA. C, Mean percentage of CR acquisition and fold difference in levels of $t T L L$ s or tTLLc mRNA expression for all treatment groups: pseudoconditioned for two pairing sessions (Ps2), conditioned for two sessions (C2), treatment with anti-tTLL siRNA for two sessions (tTLL siRNA), negative control siRNA \#6 (Nc\#6), negative control \#7 (Nc\#7), or tTLLs rescue plasmids (tTLLs Resc). Preparations treated with anti-tTLL siRNA showed no CRs and significantly reduced levels of tTLLs mRNA compared with conditioning. In contrast, preparations treated with control siRNAs showed levels of conditioning and tTLLs mRNA expression similar to untreated preparations. Treatment with the rescue plasmids also resulted in expression of CRs and tTLLs mRNA at values similar to untreated preparations. There were no differences in tTLLc among the groups.

or turtle glyceraldehyde-3-phosphate dehydrogenase (tGAPDH) expression plasmids and $12 \mathrm{~h}$ later transfected with the anti-tTLL siRNA or control siRNAs (100 nM). After a total of $25 \mathrm{~h}$, cells were examined for expression of tTLL or GAPDH by Western blotting. The anti-tTLL siRNA effectively suppressed expression of both tTLLs and tTLLc in treated cells, whereas levels of tGAPDH were unaffected (Fig. $2 A$, tTLL siRNA lane). Treatment with the negative control siRNAs failed to affect expression of either forms of tTLL or tGAPDH (Fig. 2A, Nc \#6 and Nc \#7 lanes). These data from cultured cells provided a reasonable assurance that the target gene for tTLL was selectively suppressed by our anti-tTLL siRNA oligonucleotides and could be used to examine effects of its knockdown on conditioning.

Using our battery of siRNAs, brainstem preparations were transfected by bath application of siRNA (100 nM) and Lipofectamine RNAiMAX mixed in standard oxygenated physiological saline. After $25 \mathrm{~h}$ incubation, preparations were transferred to 
normal saline and tested physiologically for their ability to be conditioned followed by subsequent analysis for tTLL expression by real-time RT-PCR. Preparations showed normal unconditioned responses and did not indicate any generalized decline in responsiveness. Data from these experiments are summarized in Figure $2 C$ in which the average percentage of CRs expressed by preparations in each treatment group (Fig. 2C, top), and the level of tTLLs and tTLLc mRNA expression are shown (Fig. 2C, bottom). As indicated above, normal conditioning for two pairing sessions resulted in CR acquisition and significant elevation in tTLLs expression compared with pseudoconditioned cases (Fig. 2C). In contrast, treatment with the anti-tTLL siRNA for two pairing sessions resulted in $0 \%$ CRs in all preparations tested and a significantly reduced fivefold expression of tTLLs compared with conditioning (Fig. $2 C, n=6$ preparations; $p<0.05$, tTLL siRNA vs C2). For comparison, preparations treated with negative control siRNAs showed levels of conditioning and tTLL expression similar to normal conditioning. Preparations treated with Negative Control \#6 siRNA showed $81 \%$ CRs and a 20 -fold increase in tTLLs (Fig. 2C, $n=3 ; p<0.0005$, Nc \#6 vs Ps2), whereas those incubated in Negative Control \#7 siRNA demonstrated $82 \%$ CRs and a 21 -fold increase in tTLLs compared with pseudoconditioning $(n=3, p<$ $0.0004, \mathrm{Nc} \# 7$ vs Ps2). There were no significant changes in tTLLc mRNA levels among any of the treatment groups examined. These data using siRNAs provide strong evidence that selective knockdown of tTLLs expression inhibits the acquisition of classical conditioning.

A powerful control for siRNA-directed inhibition of gene expression is rescue of the RNAi effect by expression of an siRNAresistant form of the targeted gene. Here, we produced a tTLL siRNA rescue plasmid for tTLLs by mutating three nucleotides (GAG to TCA) in the binding region of the tTLL siRNA. Because the tTLL gene contains sequence differences between tTLLs and tTLLc at the $5^{\prime}$ end (Sabirzhanov et al., 2007), rescue constructs could be generated specifically for the tTLLs splice variant. The tTLLs rescue plasmid was first tested for its effectiveness in resisting the anti-tTLL siRNA using an in vitro assay system (Fig. 2 B). COS- 1 cells were transfected with rescue tTLLs, or unmutated tTLLs or tTLLc expression plasmids, and then transfected with anti-tTLL siRNA. Western blot analysis showed that tTLLs was expressed only by cells treated with the rescue plasmid and not by the unmutated forms of tTLL, thereby demonstrating resistance to the inhibitory effects of the siRNA (Fig. $2 B$ ). To examine its effects on classical conditioning, the tTLLs rescue plasmid was pressure microinjected directly into the auditory and abducens nerves of brainstem preparations to allow for anterograde and retrograde transport, respectively, for selective transfection of the nerve terminals that convey the CS and the motor neurons that generate the blink response. Preparations remained in normal physiological saline for $8 \mathrm{~h}$ to allow for transport and expression of plasmid and then were incubated in anti-tTLL siRNA as described above, followed by washout and conditioning for two sessions in normal saline. Treatment with the rescue plasmid resulted in 77\% CRs, and analysis by real-time RT-PCR showed a
$\mathrm{B}$
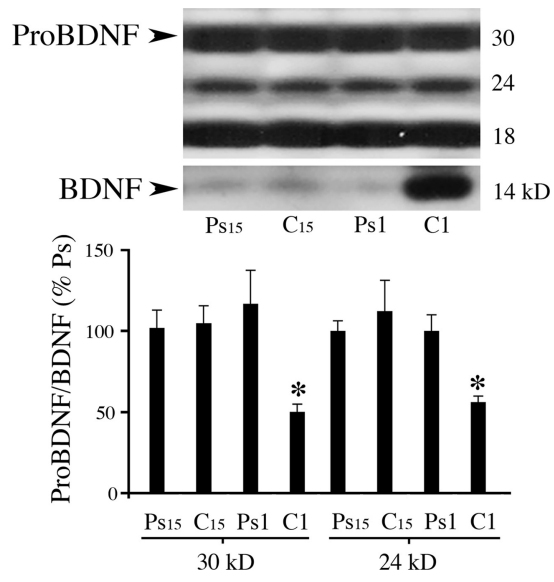

$30 \mathrm{kD}$

$24 \mathrm{kD}$

Figure 3. Conversion of proBDNF to mature BDNF occurs after the first pairing session of conditioning as shown by Western blot analysis. $A$, Significant expression of mature BDNF occurs after one complete pairing session of conditioning ( $C 1$, or after 25 min) compared with conditioning for $15 \mathrm{~min}\left(\mathrm{C}_{15}\right)$ or pseudoconditioning for the same time periods ( $\left.\mathrm{Ps}_{15}, \mathrm{Ps}_{1}\right)$. Expression of BDNF so control values after five pairing sessions ( $C 5$, or after $\sim 4 \mathrm{~h})$. $\boldsymbol{B}$, Western blots of the 30,24 , and $18 \mathrm{kDa}$ forms of proBDNF . Quantitative data of the ratio of proBDNF to BDNF for the 30 and $24 \mathrm{kDa}$ proBDNF proteins show that significant conversion

significant 16-fold increase in tTLLs mRNA expression compared with pseudoconditioned preparations (Fig. $2 C, n=4 ; p=0.0007$, tTLLs Resc vs Ps2). Together, these data provide compelling evidence that acquisition of classical conditioning requires the expression of tTLLs.

\section{Conversion of ProBDNF to BDNF is required for synaptic AMPAR delivery during acquisition of conditioning} Signaling cascades involving PKA, the $\mathrm{Ca}^{2+} /$ calmodulin-dependent protein kinases (CaMKs), and cAMP response elementbinding protein (CREB) are activated within $15 \mathrm{~min}$ of the onset of paired CS-US stimulation (Zheng and Keifer, 2009). Our previous studies also suggested that BDNF expression is a key element in the signal transduction pathway that initiates AMPAR trafficking and acquisition of CRs (Li and Keifer, 2008, 2009a). Using Western blotting, the time course of mature BDNF expression during conditioning was determined (Fig. 3). There was little expression of BDNF 15 min after the onset of the conditioning procedure similar to pseudoconditioned controls (Fig. 3A, $n=5$ per group; $p=0.55, \mathrm{C}_{15}$ vs $\left.\mathrm{Ps}_{15}\right)$. However, after one complete pairing session ( $25 \mathrm{~min}$ ), there was a significant increase in the expression of BDNF protein compared with pseudoconditioning (Fig. $3 A, p=0.002$, C1 vs Ps1). The level of BDNF expression declined to pseudoconditioned values after five pairing sessions (Fig. $3 A, p=0.37$, C5 vs Ps5). The mature form of BDNF derives from the cleavage of its precursor protein proBDNF by extracellular proteases such as plasmin or matrix metalloproteinases (Lee et al., 2001; Pang et al., 2004; Lu et al., 2005; Yang et al., 2009). ProBDNF has several different forms that are mainly composed of molecular masses at 30,24 , and $18 \mathrm{kDa}$ (Fig. $3 \mathrm{~B}$ ) that are cleaved into the $14 \mathrm{kDa}$ mature form of BDNF. During conditioning, the conversion of proBDNF to BDNF occurs by the first pairing session but not after 15 min of conditioning, as illustrated by quantitative analysis of the ratio of the 30 and $24 \mathrm{kDa}$ proBDNF proteins with mature BDNF (Fig. $3 B, n=5$ per group; $p=0.005, \mathrm{C} 1$ vs Ps1, $30 \mathrm{kDa} ; p=0.02,24 \mathrm{kDa}$ ).

During acquisition of CRs in this preparation, GluR1containing AMPARs are initially delivered to synapses followed by AMPARs containing GluR4 subunits (Mokin et al., 2007; 
A
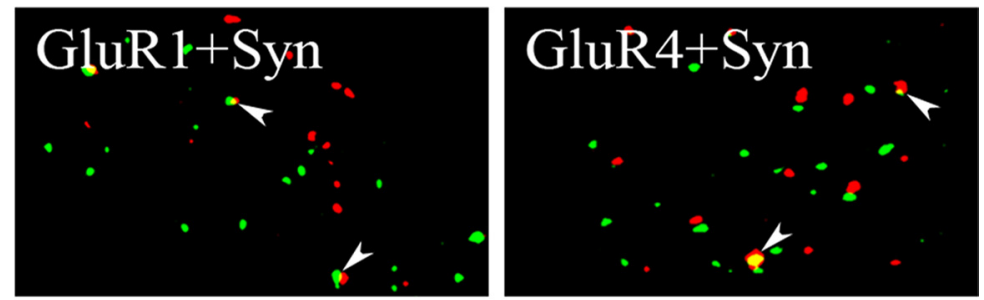

Ps2
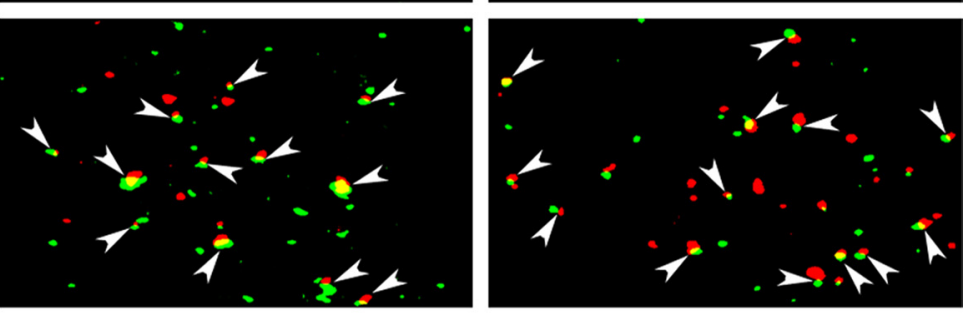

C2
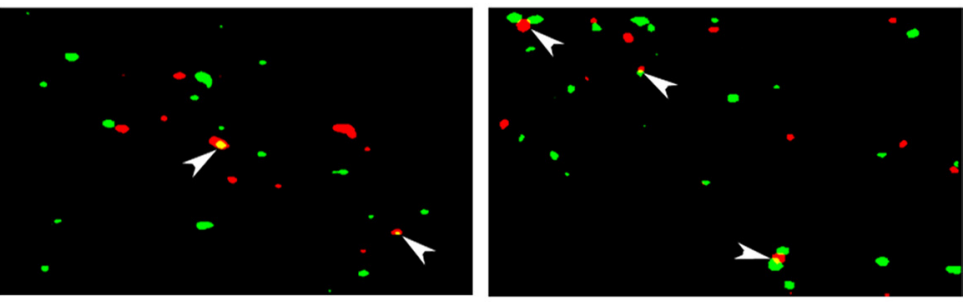

BDNF

$\mathrm{Ab}$
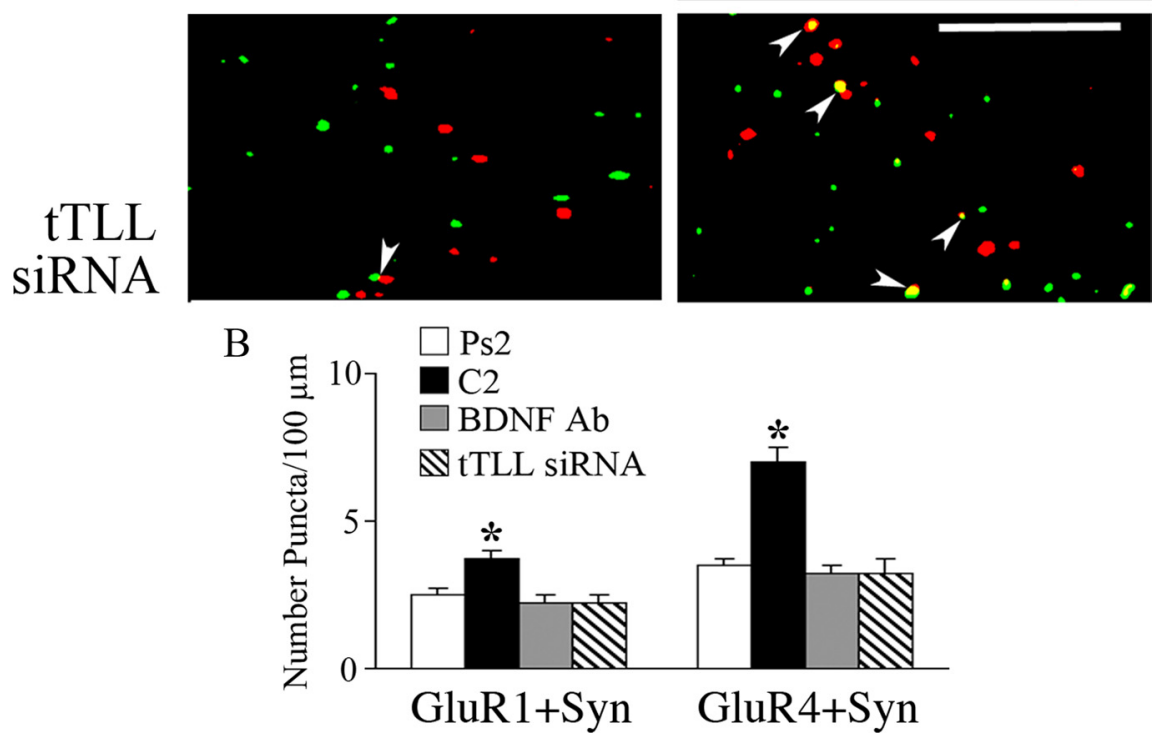

Figure 4. Synaptic incorporation of GluR1- and GluR4-containing AMPARs on abducens motor neurons observed after conditioning is inhibited by incubation of preparations in antibodies to BDNF (BDNF Ab) or anti-tTLL siRNA (tTLL siRNA). $A$, Confocal images of punctate staining for GluR1 or GluR4 AMPAR subunits (red) and the presynaptic marker synaptophysin (green) for the different treatment groups. Colocalization of AMPAR subunits with synaptophysin is indicated (arrowheads). B, Quantitative analysis of punctate staining using stereological procedures shows that the conditioning-related increase in colocalization of both GluR1 and GluR4 AMPAR subunits with synaptophysin is significantly inhibited by treatment with BDNF antibodies and tTLL siRNA. Scale bar, $2 \mu \mathrm{m}$.

GluR4+Syn, C2 vs Ps2). Elevated colocalization after conditioning is illustrated in the images by adjacent or overlapping puncta (Fig. $4 A$, arrowheads, compare C2 with Ps2). A previous study showed that coapplication of BDNF with the receptor tyrosine kinase antagonist K252a suppressed conditioning and synaptic localization of AMPARs (Li and Keifer, 2008). To further demonstrate that synaptic AMPAR incorporation is dependent on BDNF, preparations were incubated in antibodies to BDNF $(5 \mu \mathrm{g} / \mathrm{ml})$ ( $\mathrm{Li}$ and $\mathrm{Ke}-$ ifer, 2008) overnight before conditioning. This procedure suppresses BDNF expression. The results show that the treatment completely blocks conditioning to a mean of $0 \%$ CRs and significantly attenuates colocalization of GluR1 and GluR4 AMPAR subunits with synaptophysin (Fig. 4, $p<0.0001$, GluR1+Syn; $p<0.0001$, GluR4+Syn, BDNF $\mathrm{Ab}$ vs $\mathrm{C} 2$ ). Therefore, the conversion of proBDNF to mature BDNF occurs in the early stages of CR acquisition and is required for synaptic AMPAR delivery and conditioning.

\section{tTLLs cleaves proBDNF to BDNF in in vitro assays}

Because some matrix metalloproteinases are known to cleave proBDNF into its mature form and because tTLLs expression occurs at the same time as the appearance of mature BDNF during conditioning, we were interested in whether tTLLs could cleave proBDNF. In initial studies, we obtained a 40 amino acid peptide of human proBDNF (GenScript) containing the furin and plasmin cleavage site. In vitro cleavage assays showed that tTLLs, but not tTLLc, digested the peptide. We compared the known processing sites of the BMP-1/ tolloid family of metalloproteinases with the MEROPS peptidase database and the predicted site of BDNF cleavage and found that $6-8$ aa in the predicted site matched the sequences of known processing sites of BMP-1/tolloid. Because the predicted site was the same as that for furin and plasmin, we examined the ability of tTLLs to cleave proBDNF and a cleavage-resistant form of proBDNF at this site. Turtle proBDNF

Zheng and Keifer, 2008, 2009). The synaptic incorporation of AMPARs and generation of CRs requires BDNF, as shown by the imaging studies in Figure 4. Punctate staining of abducens motor neurons for GluR1 and GluR4 AMPAR subunits (red) and the presynaptic marker synaptophysin (Syn; green) was imaged using confocal microscopy, and colocalization of punctate staining, indicating synaptic localization of AMPARs, was analyzed using quantitative stereology. As shown previously, conditioning for two pairing sessions resulted in a highly significant increase in colocalization of GluR1- and GluR4-containing AMPARs with synaptophysin compared with pseudoconditioned cases (Fig. 4, $n=3$ per group; $p<0.0001$, GluR1+Syn; $p<0.0001$, was sequenced, and recombinant proteins were generated. These were incubated alone or with purified recombinant tTLLs, tTLLc, or with recombinant inactive mutants of tTLLs and tTLLc, and samples were subjected to SDS-PAGE for analysis of BDNF cleavage (Fig. 5A). The results show that only incubation of proBDNF with tTLLs was effective in cleavage of the precursor into mature BDNF (Fig. 5A, tTLLs lane). Neither tTLLc nor the mutated forms of tTLLs or tTLLc resulted in any cleavage products. To verify these results, point mutations of proBDNF were introduced into the cleavage site (by converting the $\mathrm{Arg}^{126}-\mathrm{Arg}^{127}$ to Ala-Ala). Studies have shown that this mutation is resistant to cleavage by furin and plasmin (Pang et al., 2004). Experiments 
performed in the same conditions as those above showed that this site was resistant to cleavage by tTLLs (Fig. 5B). Additional analysis of proBDNF cleavage showed that tTLLs in the same conditions cleaves proBDNF within $1 \mathrm{~h}$, as does human plasmin. However, the efficiency of cleavage by tTLLs was considerably improved in reactions performed at $25^{\circ} \mathrm{C}$ rather than $37^{\circ} \mathrm{C}$ as would be expected for turtle tissue. Therefore, these experiments demonstrate that tTLLs, but not tTLLc, converts the precursor proBDNF into mature $\mathrm{BDNF}$ in an in vitro assay system.

\section{Evidence for conversion of proBDNF to BDNF by tTLLs in brainstem preparations and effect of anti-tTLL siRNA on AMPAR trafficking}

We next determined whether tTLLs could cleave proBDNF into BDNF in our brainstem preparation in two ways. First, brainstem preparations were incubated in recombinant proteins for tTLLs or tTLLc (10 nM) without stimulation for the equivalent time period of two pairing sessions, and expression of mature BDNF was examined. As shown in Figure 6A, incubation in tTLLs resulted in significant expression of mature BDNF compared with untreated naive preparations ( $n=5$ per group; $p<0.0001$, tTLLs vs Naive) that reached $\sim 50 \%$ of the level obtained during conditioning. In contrast, incubation in tTLLc recombinant protein failed to significantly affect levels of BDNF protein ( $p=0.72$, tTLLc vs Naive). Second, because the anti-tTLL siRNA significantly attenuates conditioning and the expression of tTLLs mRNA and because evidence suggests that tTLLs cleaves proBDNF into BDNF, it was predicted that BDNF expression would be suppressed in preparations treated with the siRNA. This was found to be the case as shown in Figure 6 B. Preparations incubated in anti-tTLL siRNA (100 nM) followed by two sessions of conditioning showed $0 \%$ CRs and significantly reduced levels of BDNF compared with conditioned values (Fig. $6 B, n=5$ per group; $p=0.0002$, tTLL siRNA vs C2). Therefore, because preparations treated with tTLLs recombinant protein show significantly enhanced levels of BDNF whereas those treated with anti-tTLL siRNA show reduced levels strongly argues that tTLLs is involved in the proteolytic cleavage of proBDNF during conditioning in this preparation. Finally, given that BDNF is required for synaptic AMPAR delivery and conditioning, whether anti-tTLL siRNA treatment interferes with AMPAR trafficking during conditioning was assessed. Colocalization studies show that anti-tTLL siRNA treatment results in significantly reduced synaptic incorporation of both GluR1- and GluR4-containing AMPARs (Fig. $4 B, p<0.0001$, GluR1+Syn; $p<0.0001$, GluR4+Syn, tTLL siRNA vs $C 2$ ). Together, these findings suggest that reduced expression of $\mathrm{BDNF}$ as a result of inhibition of the proteolytic conversion of proBDNF by the anti-tTLL siRNAs results in suppression of synaptic AMPAR incorporation and conditioning.

\section{Discussion}

The primary findings of the present study are that an extracellularly secreted tolloid-like metalloproteinase, tTLLs, is transiently expressed during the early stages of in vitro eyeblink classical conditioning and functions in the conversion of proBDNF to mature BDNF that is required for synaptic delivery of AMPARs and acquisition of classical conditioning. The siRNA experiments resulting in knockdown of tTLLs and subsequent rescue by a siRNA-resistant form of tTLLs strongly support these conclusions. Specifically, the mature form of BDNF is minimally expressed in preparations treated with anti-tTLL siRNA, the synaptic incorporation of both GluR1- and GluR4-containing AMPARs is significantly reduced, and CRs fail to be acquired. Moreover, tTLLs cleaves proBDNF in in vitro cleavage assay studies, and treatment of preparations with recombinant tTLLs alone results in significant expression of mature BDNF. In contrast, a novel cytosolic form of tTLL, tTLLc, is not modulated during conditioning nor does it cleave proBDNF, and incubation of preparations in recombinant tTLLc has no effect on BDNF expression.

Previously, we described two transcripts of tTLL, tTLLs and tTLLc (Sabirzhanov et al., 2007). The difference between tTLLs and tTLLc occurs at the $5^{\prime}$ end of the transcript and influences the cellular location of the protein product. Analysis of the sequence indicates that tTLLs contains a signal sequence that specifies protein translation in the endoplasmic reticulum (ER), presumably to be secreted similar to other members of the tolloid family. In contrast, tTLLc does not contain a signal sequence and is predicted to be only in the cytoplasm or possibly secreted by a nonclassical secretory pathway independent of the ER-Golgi network. It has been shown that several proteins found in the extracellular matrix can be exported without a classical $\mathrm{N}$-terminal signal peptide. Such secreted proteins do not enter the ER and are therefore not glycosylated, although they may carry potential glycosylation motifs (Nickel, 2003), including tTLLc. Glycosylation of proteins plays an important role in modulation of enzyme activity. We also performed in vitro cleavage analysis of chordin by tTLLs and tTLLc and found that tTLLs digests chordin completely, whereas tTLLc had no effect on chordin (B. E. Sabirzhanov, J. Keifer, and T. G. Clark, unpublished observations). We can conclude from these data that tTLLc has different enzymatic activity compared with tTLLs or it is an inactive form of tTLL. The BMP-1/tolloid-like metalloproteases belong to the astacin family of metalloproteases. These enzymes are 

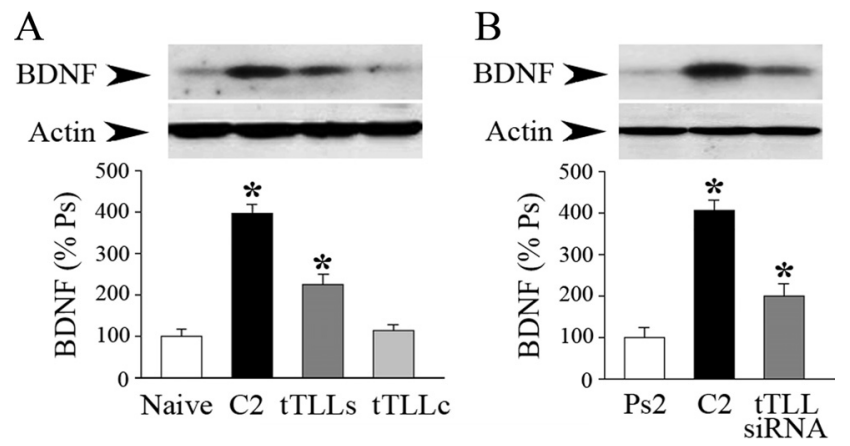

Figure 6. Brainstem preparations incubated in recombinant proteins to tTLLs generate mature BDNF and expression of BDNF during conditioning is inhibited by anti-tTLL siRNA. $A$, Western blot analysis was performed for BDNF expression in untreated (naive) and conditioned (C2) preparations and those incubated in recombinant proteins to tTLLs or tTLLc alone $(1 \mu \mathrm{g} / \mathrm{ml})$ for the same time period (the equivalent of two sessions) without stimulation. The results show that incubation in tTLLs recombinant protein generates significant levels of mature BDNF, whereas tTLLC does not. $\boldsymbol{B}$, Analysis of BDNF expression in preparations that were incubated in anti-tTLL siRNA and underwent conditioning for two pairing sessions show significantly reduced levels of mature BDNF compared with conditioning.

initially synthesized as inactive precursors with an $\mathrm{N}$-terminal prosequence. It has been shown that the proregion of astacin-like metalloproteases must be removed in the Golgi-ER for activation of enzymatic activity (Bond and Beynon, 1995). Because tTLLs has the signal peptide cleavage site, it can be secreted and activated in the ER similar to other astacin-like proteins. tTLLc does not contain this signal sequence and will be not activated in this way. The mechanisms of inactivation of BMP-1/tolloid-like metalloproteases are still unknown. However, the family of proteins known as tissue inhibitors of metalloproteinases have been shown to regulate their activity (Gomez et al., 1997).

The extracellular proteolytic cleavage of proBDNF into mature BDNF is achieved by several matrix metalloproteinases and the serine protease plasmin (Lee et al., 2001; Pang et al., 2004; Lu et al., 2005; Yang et al., 2009). This is the first study to show that a tolloid-like metalloproteinase also cleaves proBDNF and likely does so extracellularly. The tolloid/BMP-1 family of metalloproteinases are generally associated with regulation of TGF $\beta$-like proteins, including the BMPs by processing the prodomains of signaling targets, particularly during embryonic pattern formation and tissue morphogenesis (Serpe and O'Connor, 2006; Hopkins et al., 2007). TGF $\beta$-like ligands are activated by either processing extracellular inhibitors such as chordin that normally block interaction of the ligand with its receptor or by processing the $\mathrm{N}$-terminal prodomain of the ligand itself, thereby releasing it to bind to receptors. In mammals, BMP-1 and mTLL1 have been shown to cleave the extracellular antagonist of BMP signaling, chordin, thereby potentiating TGF $\beta$-like BMP signaling (Scott et al., 1999). In turtles, recombinant proteins to tTLLs, but not TLLc, cleave chordin (Sabirzhanov, Keifer, and Clark, unpublished observations), but the finding that tTLLs also processes proneurotrophins was unexpected. Cleavage is thought to occur extracellularly because tTLLs has a signal sequence at its $5^{\prime}$ end to specify secretion similar to other tolloids (Sabirzhanov et al., 2007). Interestingly, our point mutation studies resulting in a cleavage-resistant form of proBDNF demonstrate that the site of cleavage by tTLLs is after $\operatorname{Arg}^{127}$, the same as that used by furin and plasmin (Pang et al., 2004).

Similar to the TGF $\beta$-like neurotrophins, the tolloid/BMP-1s have been implicated in mechanisms of synaptic plasticity. In the marine mollusk Aplysia, the tolloid-like gene apTolloid/BMP-1 was found to be transiently regulated during nonassociative longterm sensitization training or treatment with serotonin, and mRNA was found to be particularly high in sensory neurons that mediate the sensitized response (Liu et al., 1997). However, defining a role for apTolloid/BMP-1 in the cellular mechanisms of long-term sensitization in that report was a matter of speculation. Our studies of classical conditioning using an in vitro brainstem preparation indicate that BDNF is not only required for acquisition of CRs but is a key signal transduction element in the synaptic incorporation of AMPARs during conditioning ( $\mathrm{Li}$ and Keifer, 2008, 2009a; Zheng and Keifer, 2009). Analysis of the timing of tTLLs mRNA expression by real-time RT-PCR reveals that it is dramatically increased after one and two pairing sessions at the time when CRs are acquired. The high levels of tTLLs after the first pairing session (or $25 \mathrm{~min}$ after the onset of the conditioning stimuli) also corresponds with the expression of mature BDNF protein during conditioning. However, by the fifth pairing session (or after $\sim 4 \mathrm{~h}$ ), levels of tTLLs mRNA decline to control values as do levels of BDNF protein, although asymptotic levels of CRs are expressed. High levels of tTLLs and BDNF may be required during acquisition of conditioning to induce synaptic AMPAR insertion and then once achieved other mechanisms may maintain levels of CR expression. Similar to our findings, Pang et al. (2004) found that the conversion of proBDNF into mature BDNF by activation of plasmin was an important component in the generation of late-phase LTP in hippocampal slices (Baranes et al., 1998), corroborating our conclusion that proteolytic cleavage of proneurotrophins is a significant signal transduction event in the regulation of synaptic plasticity and learning.

Our results build on a recently developed model of in vitro eyeblink classical conditioning in which BDNF is required for synaptic delivery of AMPARs and acquisition of CRs (Zheng and Keifer, 2009). Data suggest that, within minutes after the beginning of paired CS-US stimulation, PKA and CaMKIV are phosphorylated and activate the transcription factor CREB. Because mature BDNF protein expression is initiated shortly after this, one obvious target for CREB was postulated to be $B d n f$. However, levels of the precursor protein proBDNF remain unchanged as shown in this study, suggesting that it does not undergo significant regulation during conditioning, but mRNA levels were not examined. Both tTLL mRNA and BDNF protein are expressed shortly after activation of CREB (at $25 \mathrm{~min}$ rather than $15 \mathrm{~min}$ for CREB), and one possibility is that CREB, or possibly another transcription factor, directly induces transcription of the $t T l l$ gene. Once tTLLs protein is manufactured and secreted into the synaptic cleft, it cleaves proBDNF into mature BDNF. Therefore, the activity-dependent expression of the mature form of BDNF may be regulated not at the level of $B d n f$ transcription but rather at the point of the proteolytic cleavage of the precursor proBDNF. Cleavage may also be regulated by control of the secretion of extracellular proteases as suggested by Nagappan et al. (2009). In our model of learning, BDNF has been shown to activate extracellular signal-regulated kinase (ERK), which in turn induces postsynaptic AMPAR delivery that underlies conditioning (Zheng and Keifer, 2008, 2009). The intracellular activation of ERK by BDNF might proceed by way of signaling through the BDNF receptor tropomyosin-related kinase B (TrkB). Such a mechanism was proposed for a form of LTP in the mouse hippocampus. Stimulation resulted in elevation of cAMP that induced extracellular release of BDNF, followed by activation of TrkB receptors and intracellular ERK (Patterson et al., 2001). Evidence from Aplysia also suggests that TrkB is required for serotonin-induced ERK activation during long-term facilitation 
(Sharma et al., 2006). In addition to AMPAR trafficking, BDNF has known morphogenetic actions that promote axonal branching, dendritic spine growth, and increased synapse density (Tyler and Pozzo-Miller, 2001; Ji et al., 2005). Significantly, application of tissue plasminogen activator, which is involved in the formation of plasmin and conversion of proBDNF (Pang et al., 2004), to hippocampal dentate gyrus-CA3 cocultures resulted in the formation of presynaptic varicosities as well as enhanced late phase LTP (Baranes et al., 1998). In our model of classical conditioning, application of BDNF significantly increases the size of presynaptic boutons (Li and Keifer, 2009b). Therefore, the extracellular proteolytic regulation of proneurophins, such as BDNF in the synaptic cleft, has a key role in coordinating activitydependent presynaptic and postsynaptic modifications during synaptic plasticity and learning.

\section{References}

Anderson CW, Keifer J (1999) Properties of conditioned abducens nerve responses in a highly reduced in vitro brainstem preparation from the turtle. J Neurophysiol 81:1242-1250.

Baranes D, Lederfein D, Huang YY, Chen M, Bailey CH, Kandel ER (1998) Tissue plasminogen activator contributes to the late phase of LTP and to synaptic growth in the hippocampal mossy fiber pathway. Neuron 21:813-825.

Bode W, Gomis-Rüth FX, Huber R, Zwilling R, Stöcker W (1992) Structure of astacin and implications for activation of astacins and zinc-ligation of collagenases. Nature 358:164-167.

Bond JS, Beynon RJ (1995) The astacin family of metalloendopeptidases. Protein Sci 4:1247-1261.

Gomez DE, Alonso DF, Yoshiji H, Thorgeirsson UP (1997) Tissue inhibitors of metalloproteinases: structure, regulation, and biological functions. Eur J Cell Biol 74:111-122.

Hopkins DR, Keles S, Greenspan DS (2007) The bone morphogenetic protein 1/Tolloid-like metalloproteinases. Matrix Biol 26:508-523.

Ji Y, Pang PT, Feng L, Lu B (2005) Cyclic AMP controls BDNF-induced TrkB phosphorylation and dendritic spine formation in mature hippocampal neurons. Nat Neurosci 8:164-172.

Keifer J (1993) In vitro eye-blink reflex model: role of excitatory amino acid receptors and labeling of network activity with sulforhodamine. Exp Brain Res 97:239-253.

Keifer J (2001) In vitro eyeblink classical conditioning is NMDA receptor dependent and involves redistribution of AMPA receptor subunit GluR4. J Neurosci 21:2434-2441.

Keifer J (2003) In vitro classical conditioning of the turtle eyeblink reflex: approaching cellular mechanisms of acquisition. Cerebellum 2:55-61.

Keifer J, Armstrong KE, Houk JC (1995) In vitro classical conditioning of abducens nerve discharge. J Neurosci 15:5036-5048.

Lee R, Kermani P, Teng KK, Hempstead BL (2001) Regulation of cell survival by secreted proneurotrophins. Science 294:1945-1948.

Li W, Keifer J (2008) Coordinate action of pre- and postsynaptic brainderived neurotrophic factor is required for AMPAR trafficking and acquisition of in vitro classical conditioning. Neuroscience 155:686-697.

Li W, Keifer J (2009a) BDNF-induced synaptic delivery of AMPAR subunits is differentially dependent on NMDA receptors and requires ERK. Neurobiol Learn Mem 91:243-249.

Li W, Keifer J (2009b) Growth and activation of presynaptic boutons during in vitro classical conditioning and application of BDNF. Soc Neurosci Abstr 35:39.6.

Liu QR, Hattar S, Endo S, MacPhee K, Zhang H, Cleary LJ, Byrne JH, Eskin A (1997) A developmental gene (Tolloid/BMP-1) is regulated in Aplysia neurons by treatments that induce long-term sensitization. J Neurosci $17: 755-764$
Livak KJ, Schmittgen TD (2001) Analysis of relative gene expression data using real-time quantitative PCR and the -2 ddCT method. Methods 25:402-408.

Lu B, Pang PT, Woo NH (2005) The yin and yang of neurotrophin action. Nat Rev Neurosci 6:603-614.

Mokin M, Keifer J (2006) Quantitative analysis of immunofluorescent punctate staining of synaptically localized proteins using confocal microscopy and stereology. J Neurosci Methods 157:218-224.

Mokin M, Zheng Z, Keifer J (2007) Conversion of silent synapses into the active pool by selective GluR1-3 and GluR4 AMPAR trafficking during in vitro classical conditioning. J Neurophysiol 98:1278-1286.

Nagappan G, Zaitsev E, Senatorov VV Jr, Yang J, Hempstead BL, Lu B (2009) Control of extracellular cleavage of proBDNF by high frequency neuronal activity. Proc Natl Acad Sci U S A 106:1267-1272.

Nickel W (2003) The mystery of nonclassical protein secretion. A current view on cargo proteins and potential export routes. Eur J Biochem 270:2109-2119.

Pang PT, Teng HK, Zaitsev E, Woo NT, Sakata K, Zhen S, Teng KK, Yung WH, Hempstead BL, Lu B (2004) Cleavage of proBDNF by tPA/plasmin is essential for long-term hippocampal plasticity. Science 306:487-491.

Patterson SL, Pittenger C, Morozov A, Martin KC, Scanlin H, Drake C, Kandel ER (2001) Some forms of cAMP-mediated long-lasting potentiation are associated with release of BDNF and nuclear translocation of phospho-MAP kinase. Neuron 32:123-140.

Sabirzhanov BE, Keifer J, Clark TG (2007) Characterization of a novel reptilian Tolloid-like gene in the pond turtle, Pseudemys scripta elegans. Brain Res 1154:22-30.

Scott IC, Blitz IL, Pappano WN, Imamura Y, Clark TG, Steiglitz BM, Thomas CL, Maas SA, Takahara K, Cho KW, Greenspan DS (1999) Mammalian BMP-1/Tolloid-related metalloproteinases, including novel family member mammalian Tolloid-like 2, have differential enzymatic activities and distributions of expression relevant to patterning and skeletogenesis. Dev Biol 213:283-300.

Serpe M, O'Connor MB (2006) The metalloprotease Tolloid-related and its TGF-beta-like substrate Dawdle regulate Drosophila motoneuron axon guidance. Development 133:4969-4979.

Sharma SK, Sherff CM, Stough S, Hsuan V, Carew TJ (2006) A tropomyosin-related kinase B ligand is required for ERK activation, longterm synaptic facilitation, and long-term memory in Aplysia. Proc Natl Acad Sci U S A 103:14206-14210.

Tamura G, Olson D, Miron J, Clark TG (2005) Tolloid-like 1 is negatively regulated by stress and glucocorticoids. Brain Res Mol Brain Res 142:81-90.

Tyler WJ, Pozzo-Miller LD (2001) BDNF enhances quantal neurotransmitter release and increases the number of docked vesicles at the active zones of hippocampal excitatory synapses. J Neurosci 21:4249-4258.

Woo NH, Teng HK, Siao CJ, Chiaruttini C, Pang PT, Milner TA, Hempstead BL, Lu B (2005) Activation of p75NTR by pro BDNF facilitates hippocampal long-term depression. Nat Neurosci 8:1069-1077.

Yang J, Siao CJ, Nagappan G, Marinic T, Jing D, McGrath K, Chen ZY, Mark W, Tessarollo L, Lee FS, Lu B, Hempstead BL (2009) Neuronal release of proBDNF. Nat Neurosci 12:113-115.

Yiallouros I, Grosse-Berkhoff E, Stocker W (2000) The roles of Glu93 and Tyr149 in astacin-like zinc peptidases. FEBS Lett 484:224-228.

Zheng Z, Keifer J (2008) Protein kinase C-dependent and independent signaling pathways regulate synaptic GluR1 and GluR4 AMPAR subunits during in vitro classical conditioning. Neuroscience 156:872-884.

Zheng Z, Keifer J (2009) PKA has a critical role in synaptic delivery of GluR1- and GluR4-containing AMPARs during initial stages of acquisition of in vitro classical conditioning. J Neurophysiol 101:2539-2549.

Zhu D, Keifer J (2004) Pathways controlling trigeminal and auditory nerveevoked abducens eyeblink reflexes in pond turtles. Brain Behav Evol 64: 207-222. 